\title{
Multiple zeta values, Padé approximation and Vasilyev's conjecture
}

\author{
STÉPHANE FisCHLER AND TANGUY RIVOAL
}

\begin{abstract}
Sorokin gave in 1996 a new proof that $\pi$ is transcendental. It is based on a simultaneous Padé approximation problem involving certain multiple polylogarithms, which evaluated at the point 1 are multiple zeta values equal to powers of $\pi$. In this paper we construct a Padé approximation problem of the same flavour, and prove that it has a unique solution up to proportionality. At the point 1 , this provides a rational linear combination of 1 and multiple zeta values in an extended sense that turn out to be values of the Riemann zeta function at odd integers. As an application, we obtain a new proof of Vasilyev's conjecture for any odd weight, which concerns the explicit evaluation of certain hypergeometric multiple integrals, first proved by Zudilin in 2003.
\end{abstract}

Mathematics Subject Classification (2010): 11M32 (primary); 41A21, 11J72, 33C60 (secondary).

\section{Introduction}

The goal of this paper is to provide a completely new proof of Vasilyev's conjecture for any odd weight $d \geq 3$ by solving a simultaneous Padé approximation problem involving multiple polylogarithms. Before explaining in detail our approach, we provide some background. Vasilyev [21] conjectured in 1996 that, for any integers $d \geq 2$ and $n \geq 0$,

$$
J_{d, n}:=\int_{[0,1]^{d}} \frac{\prod_{j=1}^{d} x_{j}^{n}\left(1-x_{j}\right)^{n} \mathrm{~d} x_{j}}{Q_{d}\left(x_{1}, \ldots, x_{d}\right)^{n+1}} \in \mathbb{Q}+\mathbb{Q} \zeta\left(2+e_{d}\right)+\mathbb{Q} \zeta\left(4+e_{d}\right)+\cdots+\mathbb{Q} \zeta(d)
$$

where $e_{d}=0$ if $d$ is even, $e_{d}=1$ otherwise, and $Q_{1}\left(x_{1}\right):=1-x_{1}$,

$$
\begin{aligned}
Q_{d}\left(x_{1}, \ldots, x_{d}\right): & =1-Q_{d-1}\left(x_{1}, \ldots, x_{d-1}\right) x_{d}, \quad d \geq 2 \\
& =1-\left(1-\left(\cdots 1-\left(1-x_{1}\right) x_{2} \cdots\right) x_{d-1}\right) x_{d} .
\end{aligned}
$$

Both authors are partially supported by the project HAMOT (ANR 2010 BLAN-0115).

Received September 13, 2013; accepted in revised form December 26, 2013.

Published online February 2016. 
This conjecture was already known to be true for $d=2$ and $d=3$, since Beukers [3] used these integrals to get new and quick versions of Apéry's proofs [1] of the irrationality of $\zeta(2)$ and $\zeta(3)$. Beukers' integrals were generalized by Rhin and Viola in $[13,14]$ to get the best known to date irrationality measures of $\zeta(2)$ and $\zeta(3)$ (see however [26]). Vasilyev himself proved his conjecture in the cases $d=4$ and $d=5$, results which in fact led him to the conjecture. The first complete proof was given by Zudilin [25] who showed that $J_{d, n}$ is equal to a very-well-poised hypergeometric series whose value was already known to be in $\mathbb{Q}+\mathbb{Q} \zeta\left(2+e_{d}\right)+\mathbb{Q} \zeta\left(4+e_{d}\right)+\cdots+\mathbb{Q} \zeta(d)$. Two other proofs of Vasilyev's conjecture were subsequently found, one by Zlobin [23] (direct attack) and another indirect one by Krattenthaler-Rivoal [12] (limiting case of Andrews' hypergeometric identity, in the spirit of Zudilin). The fourth one, given in the present paper, is completely different since it relies on solving a simultaneous Padé approximation problem involving multiple polylogarithms.

To state this problem we need some notation. Given any finite word $\sigma$ built on a (possibly infinite) alphabet $\{a, b, \ldots\}$, we denote by $\{\sigma\}_{j}:=\sigma \sigma \cdots \sigma$ the concatenation $j$ times of $\sigma$. By convention, $\{\sigma\}_{0}=\emptyset$. We will use two alphabets, namely $\mathbb{N}^{*}=\{1,2, \ldots\}$ and $\{\ell, s\}$. We consider multiple polylogarithms in the following extended sense:

$$
\operatorname{Li}_{b_{1} b_{2} \cdots b_{p}}^{a_{1} a_{2} \cdots a_{p-1}}(z):=\sum_{k_{1} \gtrsim k_{2} \gtrsim \cdots \gtrsim k_{p} \geq 1} \frac{z^{k_{1}}}{k_{1}^{b_{1}} k_{2}^{b_{2}} \cdots k_{p}^{b_{p}}}
$$

here $|z|<1, b_{j} \in \mathbb{N}^{*}$ and $a_{j} \in\{\ell, s\}$ for all $j$. For $j=1, \ldots, p-1$, the symbol $\gtrsim \in\{>, \geq\}$ in $k_{j} \gtrsim k_{j+1}$ is determined by the following rule: it is set to $>$ if $a_{j}=s$, and to $\geq$ if $a_{j}=\ell$. In this way, $s$ stands for a strict inequality, and $\ell$ for a large one. If $a_{j}=s$ for any $j$ we obtain the usual multiple polylogarithm

$$
\operatorname{Li}_{b_{1} b_{2} \cdots b_{p}}^{\{s\}_{p-1}}(z)=\operatorname{Li}_{b_{1} b_{2} \cdots b_{p}}(z)=\sum_{k_{1}>k_{2}>\cdots>k_{p} \geq 1} \frac{z^{k_{1}}}{k_{1}^{b_{1}} k_{2}^{b_{2}} \cdots k_{p}^{b_{p}}} ;
$$

if $a_{j}=\ell$ for any $j$ we obtain the variant denoted by $\operatorname{La}_{b_{1}} b_{2} \cdots b_{p}(z)$ in [4] and by $\mathrm{Le}_{b_{1} b_{2} \cdots b_{p}}(z)$ by Ulanskiı and Zlobin, namely

$$
\mathrm{Li}_{b_{1} b_{2} \cdots b_{p}}^{\{\ell\}_{p-1}}(z)=\sum_{k_{1} \geq k_{2} \geq \cdots \geq k_{p} \geq 1} \frac{z^{k_{1}}}{k_{1}^{b_{1}} k_{2}^{b_{2}} \cdots k_{p}^{b_{p}}} .
$$

For instance, $\mathrm{Li}_{2,1}^{\ell}(z)=\sum_{k_{1} \geq k_{2} \geq 1} \frac{z^{k_{1}}}{k_{1}^{2} k_{2}}$ plays a central role in Sorokin's proof [19] that $\zeta(3)$ is irrational. Sorokin has also used in [20] the functions

$$
\operatorname{Li}_{\{1\}_{2 r+1}}^{\{s \ell\}_{r}}(1-x)=\sum_{k_{1}>k_{2} \geq k_{3}>k_{4} \geq \cdots>k_{2 r} \geq k_{2 r+1} \geq 1} \frac{(1-x)^{k_{1}}}{k_{1} k_{2} \cdots k_{2 r+1}}
$$


and

$$
\operatorname{Li}_{\{1\}_{2 r}}^{\{\ell s\}_{r-1} \ell}(1-x)=\sum_{k_{1} \geq k_{2}>k_{3} \geq k_{4}>\cdots>k_{2 r-1} \geq k_{2 r} \geq 1} \frac{(1-x)^{k_{1}}}{k_{1} k_{2} \cdots k_{2 r}},
$$

which he denoted respectively by $\varepsilon_{r}(x)$ and $\varphi_{r}(x)$. In this paper, all multiple polylogarithms $\operatorname{Li}_{b_{1} b_{2} \cdots b_{p}}^{a_{1} a_{2} \cdots a_{p-1}}(z)$ will be considered for $z \in \mathbb{C} \backslash[1, \infty)$ using analytic continuation. As usual, the integer $p$ in (1.2) is called the depth or length, and $b_{1}+\cdots+b_{p}$ is the weight. The need to consider interlacings of strict and large multiple summations for the summand of $\mathrm{Li}_{b_{1} b_{2} \cdots b_{p}}^{a_{1} a_{2} \cdots a_{p-1}}(z)$ naturally occurred to us as an effect of reducing our Padé problem to the one in Sorokin's paper [20] through differentiation and the change of variable $z \rightarrow 1-z$.

Our main result is the explicit resolution of the following simultaneous Padé approximation problem. Given integers $n, r \geq 0$, we want to find polynomials $A_{\rho, r, n}(z), B_{\rho, r, n}(z), C_{\rho, r, n}(z), D_{r, n}(z) \in \mathbb{C}[z]$, for $0 \leq \rho \leq r$, all of degree at most $n$, such that

$$
\begin{aligned}
S_{r, n}(z):= & \sum_{\rho=0}^{r}\left[A_{\rho, r, n}(z) \mathrm{Li}_{2\{1\}_{2 \rho+1}}^{\{\ell s\}_{\rho} \ell}\left(\frac{1}{z}\right)+B_{\rho, r, n}(z) \operatorname{Li}_{\{1\}_{2 \rho+2}}^{\{\ell s\}_{\rho} \ell}\left(\frac{1}{z}\right)\right. \\
& \left.+C_{\rho, r, n}(z) \operatorname{Li}_{\{1\}_{2 \rho+1}}^{\{s \ell\}_{\rho}}\left(\frac{1}{z}\right)\right]+D_{r, n}(z)=\mathcal{O}\left(\frac{1}{z^{(r+1)(n+1)}}\right) \\
U_{j, r, n}(z):= & \sum_{\rho=j}^{r} A_{\rho, r, n}(z) \operatorname{Li}_{1\{2\}_{r-\rho}}^{\{\ell\}_{r-\rho}}(1-z)+B_{j, r, n}(z) \\
= & \mathcal{O}\left((1-z)^{n+1}\right), \quad j=0, \ldots, r \\
V_{j, r, n}(z):= & \sum_{\rho=j}^{r} A_{\rho, r, n}(z) \operatorname{Li}_{\{2\}_{r-\rho+1}}^{\{\ell\}_{r-\rho}}(1-z)+C_{j, r, n}(z) \\
= & \mathcal{O}\left((1-z)^{n+1}\right), \quad j=0, \ldots, r .
\end{aligned}
$$

We will denote by $\mathcal{P}_{r, n}$ this Padé approximation problem. The various symbols $\mathcal{O}$ have the following meaning. The function $S_{r, n}(z)$ is obviously analytic at $z=\infty$ and we ask its order there to be at least $(r+1)(n+1)$. Similarly, the functions $U_{j, r, n}(z)$ and $V_{j, r, n}(z)$ are analytic at $z=1$ and we ask their orders there to be at least $n+1$. This is a mixed Padé approximation problem, namely in between type $I$ problems and type II problems. Similar mixed Padé approximation problems often occur in the Diophantine theory of (multiple) zeta values; see for instance $[10,19$, 20].

The problem $\mathcal{P}_{r, n}$ can be trivially converted into a linear algebra problem. Indeed, after noticing that $D_{r, n}(z)$ is automatically determined by the polynomials $A_{\rho, r, n}(z), B_{\rho, r, n}(z)$, and $C_{\rho, r, n}(z)$ as the polynomial part of $S_{r, n}(z)$ as $z \rightarrow \infty$, this Padé approximation problem amounts to solving a system of $3(r+1)(n+1)-1$ 
linear equations in $3(r+1)(n+1)$ unknowns (the coefficients of the polynomials $A_{\rho, r, n}(z), B_{\rho, r, n}(z)$, and $\left.C_{\rho, r, n}(z)\right)$. Hence, there is at least one non identically zero solution. Our main theorem shows that the solution is unique up to a multiplicative constant.

Theorem 1.1. For any integers $n, r \geq 0$, the function $S_{r, n}(z)$ in $\mathcal{P}_{r, n}$ is given by the following hypergeometric integral (up to a multiplicative constant), which converges for any $z \in \mathbb{C} \backslash[0,1)$ :

$$
\begin{aligned}
& S_{r, n}(z)=(-1)^{n+1} z^{(r+1)(n+1)} \\
& \times \int_{[0,1]^{2 r+3}} \frac{u_{0}^{(r+1)(n+1)-1}\left(1-u_{0}\right)^{n} \prod_{j=1}^{r+1}\left(\left(u_{j} v_{j}\right)^{(r-j+2)(n+1)-1}\left(1-u_{j}\right)^{n}\left(1-v_{j}\right)^{n}\right)}{\prod_{j=1}^{r+1}\left(\left(z-u_{0} u_{1} v_{1} \cdots u_{j-1} v_{j-1} u_{j}\right)^{n+1}\left(z-u_{0} u_{1} v_{1} \cdots u_{j} v_{j}\right)^{n+1}\right)} \mathrm{dud} \mathbf{v} .
\end{aligned}
$$

For $r=0$, the problem $\mathcal{P}_{0, n}$ and the integral for $S_{0, n}(z)$ exactly match those considered by Sorokin in [19]. However, our derivation of the integral for $S_{0, n}(z)$ is different from Sorokin's.

For any $r \geq 0$, the integral representation (1.3) provides a new proof of Vasilyev's conjecture for odd weights, by taking $z=1$ (see Section 2 for details). It would be very interesting to obtain a new proof of the infiniteness of irrational values among the $\zeta(2 r+1)$ (see [2,16]) by solving a Sorokin-type Padé problem involving multiple polylogarithms as in Theorem 1.1, as Sorokin did [19] for Apéry's theorem (see Section 6 at the end of the paper).

As alluded to above, Theorem 1.1 is based on Sorokin's proof [20] of the transcendence of $\pi$, which relies on the resolution of a simultaneous Padé approximation problem involving certain multiple polylogarithms (see Section 5.3 for details), as well as on the identity $\mathrm{Li}_{\{2\}_{r}}^{\left\{S_{r}-1\right.}(1)=\frac{\pi^{2 r}}{(2 r+1) !}$ for any integer $r \geq 1$.

The integral for $S_{r, n}(z)$ can be used to get explicit expressions of the polynomials, all of which obviously have rational coefficients. This can be done by various means, for instance one can convert the integral into the series

$$
\begin{aligned}
& S_{r, n}(z)= \\
& n ! \sum_{k_{0} \geq \cdots \geq k_{2 r+1} \geq 1} \frac{\left(k_{0}-k_{1}+1\right)_{n}\left(k_{1}-k_{2}+1\right)_{n} \cdots\left(k_{2 r}-k_{2 r+1}+1\right)_{n}\left(k_{2 r+1}-n\right)_{n}}{\prod_{j=0}^{r}\left(\left(k_{2 j}+(r-j)(n+1)\right)_{n+1}^{e_{j}}\left(k_{2 j+1}+(r-j)(n+1)\right)_{n+1}\right)} \\
& \times \frac{1}{z^{k_{0}+r(n+1)}}
\end{aligned}
$$

(where $e_{0}=2$ and $e_{j}=1$ for $j \geq 1$ ) and then use the algorithm described in [4]. 
The paper is organized as follows. In Section 2, we deduce Vasilyev's conjecture for odd values of $d$ from Theorem 1.1. In Section 3, we present a few tools needed for the proof of Theorem 1.1, in particular an iterative construction of hypergeometric multiple integrals. In Section 4, we prove an important representation formula for multiple polylogarithms and derive a few consequences useful in the resolution of $\mathcal{P}_{r, n}$. Section 5, devoted to the proof of Theorem 1.1, is decomposed in many steps. The first two steps show how to reduce the problem $\mathcal{P}_{r, n}$ to Sorokin's problem for $\pi^{2}$ (recalled in Section 5.3) and the subsequent steps complete the proof. At last we construct in Section 6 a family of integrals, containing (1.3), which enable one to prove that infinitely many odd zeta values $\zeta(2 r+1)$ are irrational $[2,16]$.

\section{A new proof of Vasilyev's conjecture for odd weights}

To deduce Vasilyev's conjecture from Theorem 1.1, we first define (when $b_{1} \geq 2$ ) extended multiple zeta values by

$$
\zeta_{b_{1} b_{2} \cdots b_{p}}^{a_{1} a_{2} \cdots a_{p-1}}:=\operatorname{Li}_{b_{1} b_{2} \cdots b_{p}}^{a_{1} a_{2} \cdots a_{p-1}}(1)=\sum_{k_{1} \gtrsim k_{2} \gtrsim \cdots \gtrsim k_{p} \geq 1} \frac{1}{k_{1}^{b_{1}} k_{2}^{b_{2}} \cdots k_{p}^{b_{p}}}
$$

with the same definition for the symbols $\gtrsim$ as in Equation (1.2). In particular, when $a_{j}=s$ for all $j$, we have the usual multiple zeta values $\zeta_{b_{1} b_{2} \cdots b_{p}}^{\{s\}_{p-1}}=\zeta\left(b_{1}, b_{2}, \ldots, b_{p}\right)$.

Then we remark that the Padé conditions for the functions $U_{j, r, n}(z)$ and $V_{j, r, n}(z)$ in $\mathcal{P}_{r, n}$ ensure that all polynomials $B_{j, r, n}(z)$ and $C_{j, r, n}(z)$ vanish at $z=1$ $(j=0, \ldots, r)$. Since multiple polylogarithms have (at most) a logarithmic singularity at $z=1$, this implies that when we take the limit $z \rightarrow 1$ in (1.3), we get

$$
\begin{gathered}
(-1)^{n+1} \int_{[0,1]^{2 r+3}} \\
\times \frac{u_{0}^{(r+1)(n+1)-1}\left(1-u_{0}\right)^{n} \prod_{j=1}^{r+1}\left(\left(u_{j} v_{j}\right)^{(r-j+2)(n+1)-1}\left(1-u_{j}\right)^{n}\left(1-v_{j}\right)^{n}\right)}{\prod_{j=1}^{r+1}\left(\left(1-u_{0} u_{1} v_{1} \cdots u_{j-1} v_{j-1} u_{j}\right)^{n+1}\left(1-u_{0} u_{1} v_{1} \cdots u_{j} v_{j}\right)^{n+1}\right)} \mathrm{dud} \mathbf{v} \\
=\sum_{\rho=0}^{r} A_{\rho, r, n}(1) \zeta_{2\{1\}_{2 \rho+1}}^{\{\ell\}_{\rho} \ell}+D_{r, n}(1)
\end{gathered}
$$

where $A_{\rho, r, n}(1)$ and $D_{r, n}(1)$ are rational numbers. Moreover, it is proved in [7, Corollaire 8] that this multiple integral is equal to $J_{2 r+3, n}$ for any integer $r \geq 0$ (see 
also Section 6 below). To complete the proof of Vasilyev's conjecture in this case, we simply need the following result, which plays the same role for us as the identity $\mathrm{Li}_{\{2\}_{r}}^{\{s\}_{r-1}}(1)=\frac{\pi^{2 r}}{(2 r+1) !}$ for Sorokin in [20].

Proposition 2.1. For any integer $k \geq 1$, we have

$$
\zeta_{2\{1\}_{2 k-1}}^{\{\ell s\}_{k-1} \ell}=\zeta_{\{2\}_{k} 1}^{\{\ell\}_{k}}=2 \zeta(2 k+1)
$$

Proof. The second equality in (2.2) is proved in Zlobin's paper [24]. To prove the first equality, which we have not found in the literature, we use the representation of (extended) multiple zeta values as Chen iterated integrals. Indeed, we have

$$
\begin{aligned}
& \zeta_{2\{1\}_{2 k-1}}^{\{\ell s\}_{k-1} \ell} \int_{\left\{0 \leq x_{2 k+1} \leq \cdots \leq x_{1} \leq 1\right\}} \frac{\mathrm{d} \mathbf{x}}{x_{1} x_{2}\left(1-x_{2}\right)\left(1-x_{3}\right) x_{4}\left(1-x_{4}\right)\left(1-x_{5}\right) \cdots x_{2 k}\left(1-x_{2 k}\right)\left(1-x_{2 k+1}\right)} \\
& =\int_{\left\{0 \leq y_{2 k+1} \leq \cdots \leq y_{1} \leq 1\right\}} \frac{\mathrm{d} \mathbf{y}}{y_{1} y_{2}\left(1-y_{2}\right) y_{3} y_{4}\left(1-y_{4}\right) y_{5} \cdots y_{2 k}\left(1-y_{2 k}\right)\left(1-y_{2 k+1}\right)}=\zeta_{\{2\}_{k} 1}^{\{\ell\}_{k}},
\end{aligned}
$$

where we have made the change of variables $x_{j}=1-y_{2 k+2-j}, j=1, \ldots, 2 k+1$.

It is in general very difficult to evaluate Sorokin-type integrals such as (1.3) (at $z=1$ ) explicitly. It is proved in [4] that they are always rational linear forms in multiple zeta values. It is much more difficult to specify which multiple zeta values have a non zero coefficient; see the papers [5,9] for some partial results in the general case. In the papers $[15,17,22]$, certain specific integrals are shown to yield only zeta values (i.e., multiple zeta values of depth 1), which is a non-trivial step. In the integral (1.3), the information is one level higher, with the cancelation of even zeta values when $z=1$.

\section{General results on multiple polylogarithms}

We gather in this section various results, useful in the proof of Theorem 1.1 but which may also be of independent interest.

\subsection{Differentiation rules for multiple polylogarithms}

In this section, we describe how to differentiate a multiple polylogarithm. To begin with, we state formulas of which the proofs are straightforward; we will use them without further mentions. The letter a denotes a finite word built on the alphabet 
$\{\ell, s\}$, the letter $\mathbf{b}$ a finite word built on the alphabet $\mathbb{N}^{*}$, and $t$ any integer $\geq 2$.

$$
\begin{aligned}
\frac{\mathrm{d}}{\mathrm{d} z} \operatorname{Li}_{1}(z) & =\frac{1}{1-z}, & \frac{\mathrm{d}}{\mathrm{d} z}\left[\operatorname{Li}_{1}\left(\frac{1}{z}\right)\right] & =\frac{1}{z(1-z)}, \\
\frac{\mathrm{d}}{\mathrm{d} z} \operatorname{Li}_{1 \mathbf{b}}^{\ell \mathbf{a}}(z) & =\frac{1}{z(1-z)} \operatorname{Li}_{\mathbf{b}}^{\mathbf{a}}(z), & \frac{\mathrm{d}}{\mathrm{d} z}\left[\operatorname{Li}_{1 \mathbf{b}}^{\ell \mathbf{a}}\left(\frac{1}{z}\right)\right] & =\frac{1}{1-z} \operatorname{Li}_{\mathbf{b}}^{\mathbf{a}}\left(\frac{1}{z}\right), \\
\frac{\mathrm{d}}{\mathrm{d} z} \mathrm{Li}_{t \mathbf{b}}^{\ell \mathbf{a}}(z) & =\frac{1}{z} \operatorname{Li}_{(t-1) \mathbf{b}}^{\ell \mathbf{a}}(z), & \frac{\mathrm{d}}{\mathrm{d} z}\left[\operatorname{Li}_{t \mathbf{b}}^{\ell \mathbf{a}}\left(\frac{1}{z}\right)\right] & =-\frac{1}{z} \mathrm{Li}_{(t-1) \mathbf{b}}^{\ell \mathbf{a}}\left(\frac{1}{z}\right), \\
\frac{\mathrm{d}}{\mathrm{d} z} \mathrm{Li}_{1 \mathbf{b}}^{s \mathbf{a}}(z) & =\frac{1}{1-z} \operatorname{Li}_{\mathbf{b}}^{\mathbf{a}}(z), & \frac{\mathrm{d}}{\mathrm{d} z}\left[\operatorname{Li}_{1 \mathbf{b}}^{\mathbf{a}}\left(\frac{1}{z}\right)\right] & =\frac{1}{z(1-z)} \operatorname{Li}_{\mathbf{b}}^{\mathbf{a}}\left(\frac{1}{z}\right), \\
\frac{\mathrm{d}}{\mathrm{d} z} \operatorname{Li}_{t \mathbf{b}}^{s \mathbf{a}}(z) & =\frac{1}{z} \operatorname{Li}_{(t-1) \mathbf{b}}^{s \mathbf{a}}(z), & \frac{\mathrm{d}}{\mathrm{d} z}\left[\operatorname{Li}_{t \mathbf{b}}^{s \mathbf{a}}\left(\frac{1}{z}\right)\right] & =-\frac{1}{z} \operatorname{Li}_{(t-1) \mathbf{b}}^{\mathbf{a}}\left(\frac{1}{z}\right) .
\end{aligned}
$$

We now state a general lemma, whose proof can be done by induction using the formulas above.

Lemma 3.1. Let $d, n \geq 0$, and $A(z) \in \mathbb{C}[z]$ be a polynomial of degree $\leq d$. Then we have

$$
\frac{\mathrm{d}^{n+1}}{\mathrm{~d} z^{n+1}}\left(A(z) \operatorname{Li}_{b_{1} b_{2} \cdots b_{p}}^{a_{1} a_{2} \cdots a_{p-1}}(z)\right)=\sum_{i=0}^{p+1} \sum_{b^{\prime}=1}^{b_{i}} \frac{\widehat{A}_{i, b^{\prime}}(z)}{z^{n+1}(1-z)^{n+1}} \operatorname{Li}_{b^{\prime} b_{i+1} b_{i+2} \cdots b_{p}}^{a_{i} a_{i+1} \cdots a_{p-1}}(z)
$$

for some polynomials $\widehat{A}_{i, b^{\prime}}(z)$ of degree $\leq d+n+1$; here we let $b_{p+1}=1$ so that in the sum there is one term corresponding to $i=p+1$, and the associated polylogarithm is equal to 1.

It is not difficult to see that in this lemma, each polynomial $\widehat{A}_{i, b^{\prime}}(z)$ depends only on $b_{1}, \ldots, b_{i-1}, a_{1}, \ldots, a_{i-1}$, and $b_{i}-b^{\prime}$. However we will not use this remark in the present paper.

Using the above relations in the same way, an analogous lemma yields polynomials $\widehat{A}_{i, b^{\prime}}^{\prime}(z)$ of degree $\leq d+n+1$ such that

$$
\frac{\mathrm{d}^{n+1}}{\mathrm{~d} z^{n+1}}\left(A(z) \operatorname{Li}_{b_{1} b_{2} \cdots b_{p}}^{a_{1} a_{2} \cdots a_{p-1}}(1 / z)\right)=\sum_{i=0}^{p+1} \sum_{b^{\prime}=1}^{b_{i}} \frac{{\widehat{A_{i, b^{\prime}}^{\prime}}}^{\prime}(z)}{z^{n+1}(1-z)^{n+1}} \operatorname{Li}_{b^{\prime} b_{i+1} b_{i+2} \cdots b_{p}}^{a_{i} a_{i+1} \cdots a_{p-1}}(1 / z) .
$$

To take advantage of vanishing conditions like the ones on $U_{j, r, n}(z)$ and $V_{j, r, n}(z)$ in the Padé problem $\mathcal{P}_{r, n}$, the following variant of Lemma 3.1 is very useful.

Lemma 3.2. Let $n \geq 0$, and $g(z)$ be a function holomorphic at $z=1$, such that $g(z)=\mathcal{O}\left((z-1)^{n+\overline{1}}\right)$ as $z \rightarrow 1$. Then we have

$$
\frac{\mathrm{d}^{n+1}}{\mathrm{~d} z^{n+1}}\left(g(z) \operatorname{Li}_{b_{1} b_{2} \cdots b_{p}}^{a_{1} a_{2} \cdots a_{p-1}}(z)\right)=\sum_{i=0}^{p+1} \sum_{b^{\prime}=1}^{b_{i}} h_{i, b^{\prime}}(z) \operatorname{Li}_{b^{\prime} b_{i+1} b_{i+2} \cdots b_{p}}^{a_{i} a_{i+1} \cdots a_{p-1}}(z)
$$

for some functions $h_{i, b^{\prime}}(z)$ holomorphic at $z=1$. As in Lemma 3.1, we let $b_{p+1}=1$ so that in the sum there is one term corresponding to $i=p+1$, and the associated polylogarithm is equal to 1 . 
In other words, no pole appears at $z=1$ if $g$ vanishes to order at least $n+1$ at this point (since polylogarithms have at most a logarithmic divergence at 1).

\subsection{An integral operator}

Sorokin solved several Padé approximation problems involving multiple polylogarithms (see [19] and [20], amongst other papers), which always lead to hypergeometric multiple integrals. We define now an integral operator intimately related to his approach (and therefore also to Theorem 1.1).

Given integers $a, b, n \geq 0$ and a function $F(z)$, we let

$$
\mathbf{H}_{a, b}^{n+1}(F)(z)=(-1)^{n+1} z^{n+1-a} \int_{0}^{1} \frac{u^{a+b-n-2}(1-u)^{n}}{(u-z)^{b}} F\left(\frac{z}{u}\right) \mathrm{d} u .
$$

The assumptions on $F$ and the properties of the function $\mathbf{H}_{a, b}^{n+1}(F)$ defined in this way are detailed in the following:

Lemma 3.3. Let $F(z)$ be holomorphic on $\mathbb{C} \backslash[0,1]$ and at $z=\infty$; denote by $\omega \geq 0$ its order of vanishing at $\infty$. Given $a, b, n \geq 0$, let $\omega^{\prime}=\omega+a+b-n-1$ and assume that $\omega^{\prime} \geq 1$.

Then $\mathbf{H}_{a, b}^{n+1}(F)$ is holomorphic on $\mathbb{C} \backslash[0,1]$ and at $z=\infty$; its order of vanishing at $\infty$ is exactly $\omega^{\prime}$. Moreover:

(i) Letting $R=\mathbf{H}_{a, b}^{n+1}(F)$, we have

$$
F(z)=\frac{1}{n !} z^{a}(1-z)^{b} R^{(n+1)}(z)
$$

(ii) If $R(z)$ is a function holomorphic on $\mathbb{C} \backslash[0,1]$ and at $z=\infty$ such that $R(\infty)=$ 0 and Equation (3.2) holds, then $R=\mathbf{H}_{a, b}^{n+1}(F)$.

We shall apply this lemma in two cases: either $F(\infty)=0$ and $a+b \geq n+1$, or $F$ is the constant function $F(z)=1$ and $a+b \geq n+2$. In both cases we have $\omega^{\prime} \geq 1$, so that $\mathbf{H}_{a, b}^{n+1}(F)$ is holomorphic on $\mathbb{C} \backslash[0,1]$ and at $z=\infty$, and $\mathbf{H}_{a, b}^{n+1}(F)(\infty)=0$.

Proof. Let $G(z)=z^{\omega} F(z)$; then $G(z)$ is holomorphic on $\mathbb{C} \backslash[0,1]$ and at $\infty$, with $G(\infty) \neq 0$. By definition of $\omega^{\prime}$ we have

$$
\mathbf{H}_{a, b}^{n+1}(F)(z)=(-1)^{n+1} z^{-\omega^{\prime}} \int_{0}^{1} \frac{u^{\omega^{\prime}-1}(1-u)^{n}}{\left(\frac{u}{z}-1\right)^{b}} G\left(\frac{z}{u}\right) \mathrm{d} u .
$$

Since $\omega^{\prime} \geq 1$ and $u / z \neq 1$ for any $u \in[0,1]$ (since $z \in \mathbb{C} \backslash[0,1]$ ), this formula shows that $\mathbf{H}_{a, b}^{n+1}(F)$ is holomorphic on $\mathbb{C} \backslash[0,1]$ and at $z=\infty$. It has order equal to $\omega^{\prime}$ at $\infty$ because $G(\infty) \neq 0$. 
To prove (i) and (ii), we perform the change of variable $x=z / u$ and deduce

$$
\mathbf{H}_{a, b}^{n+1}(F)(z)=(-1)^{n+1} \int_{z}^{\infty} \frac{(x-z)^{n}}{x^{a}(1-x)^{b}} F(x) \mathrm{d} x .
$$

Then assertions (i) and (ii) follow immediately from the following lemma, obtained from the arguments given in [18, page 60].

Lemma 3.4. Let $R, S$ be functions analytic on a neighborhood of $\infty$, with $R(\infty)=$ 0. Then

$$
\frac{1}{n !} R^{(n+1)}(z)=S(z) \Longleftrightarrow R(z)=(-1)^{n+1} \int_{z}^{\infty}(x-z)^{n} S(x) \mathrm{d} x .
$$

Note that, because of the assumptions on $R$, the left-hand side of (3.3) automatically implies the convergence of the integral on the right-hand side. But of course one must also assume the convergence of the integral for the converse. Moreover, the equivalence (3.3) is nothing but the well-known fact that the $(n+1)$-th primitive of a function (vanishing at $\infty$ ) can be recovered by the Riemann-Liouville operator defined at infinity by

$$
D_{\alpha}(f)(z)=\frac{1}{\Gamma(\alpha)} \int_{\infty}^{z}(z-x)^{\alpha-1} f(x) \mathrm{d} x .
$$

For Diophantine applications the value $\mathbf{H}_{a, b}^{n+1}(F)(1)$ is often the most interesting one; conditions for this value to exist are given by the following lemma, whose proof is straightforward.

Lemma 3.5. Assume that $b \leq n+1$ and $F(z)$ has (at most) a power of logarithm divergence as $z \rightarrow 1$, with $z \in \mathbb{C} \backslash[0,1]$ (i.e., $F(z)$ is locally a polynomial in $\log (z-1)$ with coefficients holomorphic at 1$)$. Then $\mathbf{H}_{a, b}^{n+1}(F)(z)$ has also (at most) a power of logarithm divergence as $z \rightarrow 1$, with $z \in \mathbb{C} \backslash[0,1]$.

Moreover, if in addition $b \leq n$ then $\mathbf{H}_{a, b}^{n+1}(F)(z)$ has a finite limit as $z \rightarrow$ 1 , with $z \in \mathbb{C} \backslash[0,1]$, and this limit is given by taking $z=1$ in the integral representation of Equation (3.1), which is then convergent.

In Padé approximation problems with multiple polylogarithms, multiple integrals appear by applying successively integral operators $\mathbf{H}_{a, b}^{n+1}$ with various parameters. We shall write $\mathbf{H}_{a, b}^{n+1} \mathbf{H}_{a^{\prime}, b^{\prime}}^{n^{\prime}+1}$ for $\mathbf{H}_{a, b}^{n+1} \circ \mathbf{H}_{a^{\prime}, b^{\prime}}^{n^{\prime}+1}$, so that $\mathbf{H}_{a, b}^{n+1} \mathbf{H}_{a^{\prime}, b^{\prime}}^{n^{\prime}+1}(F)=$ $\mathbf{H}_{a, b}^{n+1}\left(\mathbf{H}_{a^{\prime}, b^{\prime}}^{n^{\prime}+1}(F)\right)$. We shall consider in Subsections 5.4 and 5.5 multiple integrals of the form

$$
\mathbf{H}_{a_{1}, b_{1}}^{n_{1}+1} \mathbf{H}_{a_{2}, b_{2}}^{n_{2}+1} \cdots \mathbf{H}_{a_{p}, b_{p}}^{n_{p}+1}(\mathbf{1})
$$

where the $a_{j}, b_{j}, n_{j}$ are non-negative integers and $\mathbf{1}$ denotes the function equal to 1 on $\mathbb{C} \backslash[0,1]$; such integrals appear in Sorokin's papers (e.g., [19] and [20]). Lemma 3.3 gives conditions on the parameters that ensure that this integral expression is 
holomorphic on $\mathbb{C} \backslash[0,1]$ and at $z=\infty$, and Lemma 3.5 plays the analogous role for the behaviour at $z=1$.

In the proof of Theorem 1.1 we shall use the following result which describes the behaviour of this integral operator under the change of variable $z \mapsto 1-z$.

Lemma 3.6. For any integers $a_{j}, b_{j}, n_{j}, j=1, \ldots, p$ such that $\mathbf{H}_{a_{1}, b_{1}}^{n_{1}+1} \mathbf{H}_{a_{2}, b_{2}}^{n_{2}+1} \ldots$ $\cdots \mathbf{H}_{a_{p}, b_{p}}^{n_{p}+1}(\mathbf{1})$ is holomorphic on $\mathbb{C} \backslash[0,1]$ and at $\infty$, we have

$\mathbf{H}_{a_{1}, b_{1}}^{n_{1}+1} \mathbf{H}_{a_{2}, b_{2}}^{n_{2}+1} \cdots \mathbf{H}_{a_{p}, b_{p}}^{n_{p}+1}(\mathbf{1})(1-z)=(-1)^{p+n_{1}+n_{2}+\cdots+n_{p}} \mathbf{H}_{b_{1}, a_{1}}^{n_{1}+1} \mathbf{H}_{b_{2}, a_{2}}^{n_{2}+1} \cdots \mathbf{H}_{b_{p}, a_{p}}^{n_{p}+1}(\mathbf{1})(z)$

for all $z \in \mathbb{C} \backslash[0,1]$.

Proof. This is a consequence of the following fact. Given $f(z)$, we set $f^{\partial}(z):=$ $f(1-z)$. Then

$$
R(z)=\mathbf{H}_{a, b}^{n+1}(S)(z) \Longleftrightarrow R^{\partial}(z)=(-1)^{n+1} \mathbf{H}_{b, a}^{n+1}\left(S^{\partial}\right)(z) .
$$

This equivalence results from Lemma 3.3:

$$
S(z)=\frac{1}{n !} z^{a}(1-z)^{b} R^{(n+1)}(z) \Longleftrightarrow S(1-z)=\frac{(-1)^{n+1}}{n !} z^{b}(1-z)^{a}(R(1-z))^{(n+1)} .
$$

\subsection{Functional linear independence of polylogarithms}

The extended multiple polylogarithms defined in the introduction are very useful to state and prove our result, but they are not really new functions: they are linear combinations over $\mathbb{Z}$ of usual multiple polylogarithms (corresponding to $\alpha_{1}=\ldots=\alpha_{p-1}=s$ in (1.2)). This follows from the following elementary relation (which is the starting point of [4]):

$$
\operatorname{Li}_{b_{1} b_{2} \cdots b_{p}}^{a_{1} \cdots a_{j-1} \ell a_{j+1} \cdots a_{p-1}}(z)=\operatorname{Li}_{b_{1} b_{2} \cdots b_{p}}^{a_{1} \cdots a_{j-1} s a_{j+1} \cdots a_{p-1}}(z)+\operatorname{Li}_{b_{1} \cdots b_{j-1} b^{\prime} b_{j+2} \cdots b_{p}}^{a_{1} \cdots a_{j-1} a_{j+1} \cdots a_{p-1}}(z)
$$

where $b^{\prime}=b_{j}+b_{j+1}$.

In the proof of Theorem 1.1 we shall use the following result.

Lemma 3.7. For any $k \geq 0$, let $\mathbf{a}_{k}$ be a word on the alphabet $\{\ell, s\}$ of length $k-1$, with $\mathbf{a}_{1}=\mathbf{a}_{0}=\emptyset$. Then the polylogarithms $\operatorname{Li}_{\{1\}_{k}}^{\mathbf{a}_{k}}(1 / z)$, for $k \geq 0$, are linearly independent over the field $\mathcal{M}_{0}$ of functions meromorphic at 1 .

Proof. To begin with, let us consider for any $p \geq 0$ the set $\mathcal{F}_{p}$ of all functions analytic on $\mathbb{C} \backslash[0,1]$ that can be written as $\sum_{i=0}^{p} h_{i}(z)\left(\log \left(1-\frac{1}{z}\right)\right)^{i}$ where $h_{0}(z), \ldots$, $h_{p}(z)$ are functions holomorphic on $\mathbb{C} \backslash[0,1]$ and at $z=1$. Of course all functions holomorphic on $\mathbb{C} \backslash[0,1]$ and at $z=1$ belong to $\mathcal{F}_{0}$, and $\operatorname{Li}_{1}(1 / z)=-\log \left(1-\frac{1}{z}\right)$ 
belongs to $\mathcal{F}_{1}$. We claim that for any $p \geq 0$, for any $\alpha_{1}, \ldots, \alpha_{p-1} \in\{\ell, s\}$ and any $b_{1}, \ldots, b_{p} \geq 1$, we have

$$
\mathrm{Li}_{b_{1} b_{2} \cdots b_{p}}^{a_{1} \cdots a_{p-1}}(1 / z) \in \mathcal{F}_{p} .
$$

In other words, all multiple polylogarithms of depth $p$ belong to $\mathcal{F}_{p}$. This claim can be proved using monodromy, but we shall prove it directly by induction on the weight $b_{1}+\cdots+b_{p}$. We have already noticed that it holds if $b_{1}+\cdots+b_{p} \leq 1$. Now remark that if $f$ is analytic on $\mathbb{C} \backslash[0,1]$ and $g \in \mathcal{F}_{p}$ is such that $f^{\prime}(z)=\frac{-1}{z} g(z)$ then $f \in \mathcal{F}_{p}$, because $\mathcal{F}_{p}$ is stable under primitivation and products with functions holomorphic at 1 . On the other hand, if $f^{\prime}(z)=\frac{1}{1-z} g(z)$ or $f^{\prime}(z)=\frac{1}{z(1-z)} g(z)$ then $f \in \mathcal{F}_{p+1}$. Using the differentiation rules for polylogarithms stated at the beginning of Subsection 3.1, this proves the claim.

Now assume that for some $k \geq 1$ the function $\mathrm{Li}_{\{1\}_{k}}^{\mathbf{a}_{k}}(1 / z)$ is a linear combination over $\mathcal{M}_{0}$ of the $\operatorname{Li}_{\{1\}_{j}}^{\mathbf{a}_{j}}(1 / z)$ for $0 \leq j \leq k-1$. Using the claim this implies $\mathrm{Li}_{\{1\}_{k}}^{\mathbf{a}_{k}}(1 / z) \in \mathcal{F}_{k-1}$. Now applying Equation (3.4) as many times as needed one can write $\operatorname{Li}_{\{1\}_{k}}^{\mathbf{a}_{k}}(1 / z)-\mathrm{Li}_{\{1\}_{k}}^{\{s\}_{k-1}}(1 / z)$ as a $\mathbb{Z}$-linear combination of extended multiple polylogarithms of depth $k-1$; applying the claim again proves that $\operatorname{Li}_{\{1\}_{k}}^{\{s\}_{k-1}}(1 / z)=$ $(-1)^{k}\left(\log \left(1-\frac{1}{z}\right)\right)^{k}$ belongs to $\mathcal{F}_{k-1}$ (this identity belongs to the folklore and is readily proved by induction and differentiation). But this provides a non-trivial linear relation, with coefficients holomorphic at 1 , between powers of the function $\log \left(1-\frac{1}{z}\right)$. This is impossible since $\log (z)$ is transcendental over the field of functions meromorphic at the origin. This contradiction concludes the proof of Lemma 3.7.

\section{Weight functions of multiple polylogarithms}

In this section we study the weight functions of multiple polylogarithms and compute some of them. This part is at the heart of the proof of Theorem 1.1, since weights obey the same derivation rules as the corresponding polylogarithms (see below).

Throughout this section, we consider non-empty words $\mathbf{b}$. It is well-known that usual multiple polylogarithms $\operatorname{Li}_{\mathbf{b}}^{a}(z)$ (with $\boldsymbol{a}=s s \cdots s$ ) can be analytically continued to the cut plane $\mathbb{C} \backslash[1,+\infty)$. They vanish at $z=0$ and their growth as $z \rightarrow \infty$ is at most a power of $\log (z)$, with $0<\arg (z)<2 \pi$. Moreover, the function defined on the cut by

$$
\lim _{y \rightarrow 0+}\left[\operatorname{Li}_{\mathbf{b}}^{s s \cdots s}(x+i y)-\mathrm{Li}_{\mathbf{b}}^{s s \cdots s}(x-i y)\right]
$$

is $C^{\infty}$ on $(1,+\infty)$ with at most a (power of) logarithm singularity at $x=1$ and $x=\infty$. All these properties also hold for $\operatorname{Li}_{\mathbf{b}}^{a}(z)$ for any word a because such functions are simply linear combinations with rational coefficients of the $\mathrm{Li}_{\mathbf{b}}^{s s \cdots s}(z)$ (using repeatedly Equation (3.4) above). 
As an (important) application, we prove the following:

Lemma 4.1. For any fixed $z \in \mathbb{C} \backslash[0,1]$, any $\mathbf{a}$ and any $\mathbf{b} \neq \emptyset$, we have

$$
\operatorname{Li}_{\mathbf{b}}^{a}\left(\frac{1}{z}\right)=\int_{0}^{1} \frac{\omega_{\mathbf{b}}^{a}(x)}{z-x} \mathrm{~d} x
$$

where

$$
\omega_{\mathbf{b}}^{a}(x):=\frac{1}{2 i \pi} \lim _{y \rightarrow 0+}\left[\operatorname{Li}_{\mathbf{b}}^{a}\left(\frac{1}{x}+i y\right)-\operatorname{Li}_{\mathbf{b}}^{a}\left(\frac{1}{x}-i y\right)\right] \in L^{1}([0,1]) .
$$

The weight function $\omega_{\mathbf{b}}^{a}(x)$ is $C^{\infty}$ on $(0,1)$, with at most (power of) logarithm singularities at $x=0$ and $x=1$.

Proof. For any fixed $z \in \mathbb{C} \backslash[1,+\infty)$, let us consider the Cauchy representation formula

$$
\operatorname{Li}_{\mathbf{b}}^{a}(z)=\frac{z}{2 i \pi} \int_{\mathcal{C}} \frac{\mathrm{Li}_{\mathbf{b}}^{a}(t)}{t(t-z)} \mathrm{d} t,
$$

where $\mathcal{C}$ is any simple closed curve surrounding $z$ and not crossing the cut $[1,+\infty)$. We can deform $\mathcal{C}$ to a simple closed curve defined as follows: given $\varepsilon>0$ and $R>0$ (such that $|z|<R$ ), we glue together two straight lines $[1+i \varepsilon, R+i \varepsilon]$, $[1-i \varepsilon, R-i \varepsilon]$, a semi-circle of center 1 and diameter $[1-i \varepsilon, 1+i \varepsilon]$ and an arc of circle of center 0 passing through $R+i \varepsilon$ and $R-i \varepsilon$ (both arcs not crossing $[1,+\infty))$. The analytic properties of $\mathrm{Li}_{\mathbf{b}}^{a}(z)$ are such that we can let $\varepsilon \rightarrow 0$ and $R \rightarrow \infty$ to get the representation

$$
\begin{aligned}
\operatorname{Li}_{\mathbf{b}}^{a}(z) & =z \int_{1}^{\infty} \frac{\omega_{\mathbf{b}}^{a}(1 / t)}{t(t-z)} \mathrm{d} t \\
& \left.=z \int_{0}^{1} \frac{\omega_{\mathbf{b}}^{a}(x)}{1-z x} \mathrm{~d} x \quad \text { (by letting } x=1 / t\right),
\end{aligned}
$$

where $\omega_{\mathbf{b}}^{\boldsymbol{a}}(x)$ is defined by (4.2). We obtain (4.1) by changing $z$ to $1 / z$.

(This proof is not specific to multiple polylogarithms. Such weighted integral representations are known as Stieltjes representations; see [11, page 591, Theorem 12.10d].)

We note two important consequences of the expression (4.2) for $\omega_{\mathbf{b}}^{a}(x)$. To begin with, if

$$
\frac{\mathrm{d}}{\mathrm{d} z}\left[\operatorname{Li}_{\mathbf{b}}^{a}\left(\frac{1}{z}\right)\right]=R(z) \operatorname{Li}_{\mathbf{b}^{\prime}}^{a^{\prime}}\left(\frac{1}{z}\right)
$$

then

$$
\frac{\mathrm{d}}{\mathrm{d} x} \omega_{\mathbf{b}}^{a}(x)=R(x) \omega_{\mathbf{b}^{\prime}}^{\boldsymbol{a}^{\prime}}(x)
$$


where the function $R(z)$ is one of $-\frac{1}{z}, \frac{1}{1-z}$ and $\frac{1}{z(1-z)}$ (see Subsection 3.1). In other words, weights obey the same derivation rules as the corresponding polylogarithms. This observation will be crucial in Subsection 5.1. Moreover, we also remark that if the value $\mathrm{Li}_{\mathbf{b}}^{a}(1)$ is finite, then $\omega_{\mathbf{b}}^{a}(1)=0$.

Lemma 4.2. For any $x \in(0,1)$ and any integer $k \geq 0$, we have

$$
\begin{aligned}
& \omega_{\{1\}_{2 k}}^{\{\ell s\}_{k-1} \ell}(x)=\mathrm{Li}_{\{1\}_{2 k-1}}^{\{s \ell\}_{k-1}}(x), \\
& \omega_{\{1\}_{2 k+1}}^{\{s \ell\}_{k}}(x)=\mathrm{Li}_{\{1\}_{2 k}}^{\{\ell s\}_{k-1} \ell}(x),
\end{aligned}
$$

and

$$
\begin{aligned}
\omega_{2\{1\}_{2 k+1}}^{\{\ell s\}_{k} \ell}(x)= & \sum_{j=0}^{k} \operatorname{Li}_{1\{2\}_{j}}^{\{\ell\}_{j}}(1-x) \operatorname{Li}_{\{1\}_{2 k-2 j+1}}^{\{s \ell\}_{k-j}}(x) \\
& +\sum_{j=1}^{k+1} \operatorname{Li}_{\{2\}_{j}}^{\{\ell\}_{j-1}}(1-x) \operatorname{Li}_{\{1\}_{2 k-2 j+2}}^{\{\ell s\}_{k-j} \ell}(x) \\
= & -\operatorname{Li}_{2\{1\}_{2 k}}^{\{s \ell\}_{k}}(x)+\operatorname{Li}_{\{2\}_{k+1}}^{\{\ell\}_{k}}(1) .
\end{aligned}
$$

Proof. Equations (4.3) and (4.4) are readily checked by expanding $\frac{1}{z-x}=$ $\sum_{n=0}^{\infty} \frac{x^{n}}{z^{n+1}}$ in the integral (4.1). To prove (4.5), we remark that both sides differentiate to the same function $-\frac{1}{x} \omega_{\{1\}_{2 k+2}}^{\{\ell s\}_{k} \ell}(x)=-\frac{1}{x} \mathrm{Li}_{\{1\}_{2 k+1}}^{\{s \ell\}_{k}}(x)$, since all functions but this precise one are killed by telescoping when differentiating the right-hand side of (4.5). It follows that the functions on both sides of (4.5) differ only by a constant. This constant must be 0 because both sides vanish at $x=1$ (see the remark just before Lemma 4.2). The same argument yields also

$$
\omega_{2\{1\}_{2 k+1}}^{\{\ell s\}_{k} \ell}(x)=-\int \frac{1}{x} \operatorname{Li}_{\{1\}_{2 k+1}}^{\{s \ell\}_{k}}(x) \mathrm{d} x=-\operatorname{Li}_{2\{1\}_{2 k}}^{\{s \ell\}_{k}}(x)+C_{k}
$$

for some constant $C_{k}$. This constant is seen to be equal to $\operatorname{Li}_{\{2\}_{k+1}}^{\{\ell\}_{k}}$ (1) by taking $x=0$ in (4.5). This proves (4.6), and concludes the proof of Lemma 4.2.

In the setting of the Padé problem $\mathcal{P}_{r, n}$, we define the function

$$
P_{r, n}(z)=\sum_{\rho=0}^{r}\left[A_{\rho, r, n}(z) \omega_{2\{1\}_{2 \rho+1}}^{\{\ell s\}_{\rho} \ell}(z)+B_{\rho, r, n}(z) \omega_{\{1\}_{2 \rho+2}}^{\{\ell s\}_{\rho} \ell}(z)+C_{\rho, r, n}(z) \omega_{\{1\}_{2 \rho+1}}^{\{s \ell\}_{\rho}}(z)\right]
$$

obtained from $S_{r, n}$ by replacing every polylogarithm with its weight (see Lemma 4.4 below). By (4.3), (4.4) and (4.6), this function $P_{r, n}$ is analytic on the disk $|z|<1$, with a (power of) logarithm singularity at $z=1$. In particular, it is in $L^{1}([0,1])$. The following lemma is an immediate consequence of (4.3), (4.4), (4.5) and the definition of $U_{j, r, n}(z)$ and $V_{j, r, n}(z)$. As in the rest of the paper, we continue analytically all polylogarithms to $\mathbb{C} \backslash[1,+\infty)$. 
Lemma 4.3. For any $z \in \mathbb{C} \backslash[1,+\infty)$,

$$
P_{r, n}(z)=\sum_{j=0}^{r}\left[U_{j, r, n}(z) \operatorname{Li}_{\{1\}_{2 j+1}}^{\{s \ell\}_{j}}(z)+V_{j, r, n}(z) \operatorname{Li}_{\{1\}_{2 j}}^{\{\ell s\}_{j-1} \ell}(z)\right] .
$$

We conclude this section with the precise connection between $P_{r, n}(z)$ and $S_{r, n}(z)$.

Lemma 4.4. In the setting of the Padé problem $\mathcal{P}_{r, n}$, for any $z \in \mathbb{C} \backslash[0,1]$ we have

$$
S_{r, n}(z)=\int_{0}^{1} \frac{P_{r, n}(x)}{z-x} \mathrm{~d} x .
$$

Proof. By definition of $S_{r, n}(z)$ and Lemma 4.1 , for any $z \in \mathbb{C} \backslash[0,1]$ we have

$$
\begin{aligned}
S_{r, n}(z)= & \sum_{\rho=0}^{r}\left[A_{\rho, r, n}(z) \int_{0}^{1} \frac{\omega_{2\{1\}_{2 \rho+1}}^{\{\ell s\}_{\rho} \ell}}{z-x} \mathrm{~d} x+B_{\rho, r, n}(z) \int_{0}^{1} \frac{\omega_{\{1\}_{2 \rho+2}}^{\{\ell s\}_{\rho} \ell}(x)}{z-x} \mathrm{~d} x\right. \\
& \left.+C_{\rho, r, n}(z) \int_{0}^{1} \frac{\omega_{\{1\}_{2 \rho+1}}^{\{s \ell\}_{\rho}}(x)}{z-x} \mathrm{~d} x\right]+D_{r, n}(z) \\
= & \int_{0}^{1} \frac{P_{r, n}(x)}{z-x} \mathrm{~d} x+\sum_{\rho=0}^{r} \int_{0}^{1}\left[\frac{A_{\rho, r, n}(z)-A_{\rho, r, n}(x)}{z-x} \omega_{2\{1\}_{2 \rho+1}}^{\{\ell\}_{\rho} \ell}(x)\right. \\
& +\frac{B_{\rho, r, n}(z)-B_{\rho, r, n}(x)}{z-x} \omega_{\{1\}_{2 \rho+2}}^{\{\ell s\}_{\rho} \ell}(x) \\
& \left.+\frac{C_{\rho, r, n}(z)-C_{\rho, r, n}(x)}{z-x} \omega_{\{1\}_{2 \rho+1}}^{\{s \ell\}_{\rho}}(x)\right] \mathrm{d} x+D_{r, n}(z) .
\end{aligned}
$$

Hence,

$$
S_{r, n}(z)=\int_{0}^{1} \frac{P_{r, n}(x)}{z-x} \mathrm{~d} x+\operatorname{polynomial}(z) .
$$

But, as $z \rightarrow \infty, S_{r, n}(z)=\mathcal{O}(1 / z)$ and $\int_{0}^{1} \frac{P_{r, n}(x)}{z-x} \mathrm{~d} x \rightarrow 0$ (because $P_{r, n}(x) \in$ $L^{1}([0,1])$, as noticed above). Therefore, the polynomial in (4.7) is identically 0 and this completes the proof of Lemma 4.4.

\section{Resolution of the Padé problem $\mathcal{P}_{r, n}$}

In this section we prove Theorem 1.1, using the tools of Sections 3 and 4. Starting with a solution $S_{r, n}(z)$ of the Padé problem $\mathcal{P}_{r, n}$, we apply the differential operator $\frac{z^{n+1}}{n !}\left(\frac{\mathrm{d}}{\mathrm{d} z}\right)^{n+1}$ and prove in Subsections 5.1 and 5.2 that the resulting function is a solution of another Padé approximation problem, denoted by $\mathcal{Q}_{r, n}$ and stated in Subsection 5.3. Then we observe in Subsection 5.3 that $\mathcal{Q}_{r, n}$ is nothing but Sorokin's 
problem [20] for $\pi^{2}$, denoted by $\mathcal{R}_{r, n}$, up to a change of variable $z \mapsto 1-z$. Since Sorokin has proved that $\mathcal{R}_{r, n}$ has a unique solution up to proportionality, the same result holds for $\mathcal{Q}_{r, n}$ and $\mathcal{P}_{r, n}$.

To conclude the proof of Theorem 1.1, we deduce in Subsections 5.4 and 5.5 the integral representation (1.3) of $S_{r, n}(z)$ from Sorokin's integral representation of the solution of $\mathcal{R}_{r, n}$, using the integral operator introduced in Subsection 3.2.

\subsection{First reduction}

Let $S_{r, n}(z)$ be a solution of the Padé problem $\mathcal{P}_{r, n}$. By Lemma 3.1, there exist some polynomials $\check{A}_{\rho, r, n}(z), \check{B}_{\rho, r, n}(z)$ and $\check{C}_{r, n}(z)$ of degree $\leq 2 n+1$ such that

$$
\begin{aligned}
\widehat{S}_{r, n}(z):= & \frac{z^{n+1}}{n !} S_{r, n}^{(n+1)}(z)=\sum_{\rho=0}^{r}\left[\frac{\check{A}_{\rho, r, n}(z)}{(1-z)^{n+1}} \operatorname{Li}_{\{1\}_{2 \rho+2}}^{\{\ell\}_{\rho} \ell}\left(\frac{1}{z}\right)\right. \\
& \left.+\frac{\check{B}_{\rho, r, n}(z)}{(1-z)^{n+1}} \mathrm{Li}_{\{1\}_{2 \rho+1}}^{\left\{\ell_{\rho}\right.}\left(\frac{1}{z}\right)\right]+\frac{\check{C}_{r, n}(z)}{(1-z)^{n+1}} \\
= & \mathcal{O}\left(\frac{1}{z^{(r+1)(n+1)}}\right) .
\end{aligned}
$$

As in Section 4 we consider the function $P_{r, n}(z)$ defined by

$P_{r, n}(z)=\sum_{\rho=0}^{r}\left[A_{\rho, r, n}(z) \omega_{2\{1\}_{2 \rho+1}}^{\{\ell s\}_{\rho} \ell}(z)+B_{\rho, r, n}(z) \omega_{\{1\}_{2 \rho+2}}^{\{\ell s\}_{\rho} \ell}(z)+C_{\rho, r, n}(z) \omega_{\{1\}_{2 \rho+1}}^{\{s \ell\}_{\rho}}(z)\right]$.

Since it is obtained from $S_{r, n}$ by replacing each polylogarithm by its weight, it obeys the same derivation rules (see the remark before Lemma 4.2). This implies that

$$
\begin{aligned}
\widehat{P}_{r, n}(z) & :=\frac{z^{n+1}}{n !} P_{r, n}^{(n+1)}(z) \\
& =\sum_{\rho=0}^{r}\left[\frac{\check{A}_{\rho, r, n}(z)}{(1-z)^{n+1}} \omega_{\{1\}_{2 \rho+2}}^{\{l s\}_{\rho} \ell}(z)+\frac{\check{B}_{\rho, r, n}(z)}{(1-z)^{n+1}} \omega_{\{1\}_{2 \rho+1}}^{\{s\}_{\rho}}(z)\right] \\
& =\sum_{\rho=0}^{r}\left[\frac{\check{A}_{\rho, r, n}(z)}{(1-z)^{n+1}} \mathrm{Li}_{\{1\}_{2 \rho+1}}^{\{s \ell\}_{\rho}}(z)+\frac{\check{B}_{\rho, r, n}(z)}{(1-z)^{n+1}} \mathrm{Li}_{\{1\}_{2 \rho}}^{\{\ell s\}_{\rho-1} \ell}(z)\right]
\end{aligned}
$$

with the same polynomials $\check{A}_{\rho, r, n}(z)$ and $\check{B}_{\rho, r, n}(z)$; here we have used Equations (4.3) and (4.4) in Lemma 4.2 to compute the weights.

Now, by Lemmas 3.2, 4.3 and the Padé conditions at $z=1$ in $\mathcal{P}_{r, n}$ for $U_{j, r, n}$ and $V_{j, r, n}$, the function $\widehat{P}_{r, n}(z)$ is necessarily of the form

$$
\widehat{P}_{r, n}(z)=\sum_{j=0}^{r}\left[h_{2 j+1}(z) \operatorname{Li}_{\{1\}_{2 j+1}}^{\{s \ell\}_{j}}(z)+h_{2 j}(z) \operatorname{Li}_{\{1\}_{2 j}}^{\{\ell s\}_{j-1} \ell}(z)\right]
$$


for some functions $h_{j}$ holomorphic at $z=1$. We have obtained two expressions for $\widehat{P}_{r, n}(z)$, namely Equations (5.2) and (5.3). Using Lemma 3.7 they have to coincide, that is $\frac{\check{A}_{\rho, r, n}(z)}{(1-z)^{n+1}}=h_{2 \rho+1}(z)$ and $\frac{\check{B}_{\rho, r, n}(z)}{(1-z)^{n+1}}=h_{2 \rho}(z)$ for any $\rho=0, \ldots, r$. Therefore $(1-z)^{n+1}$ divides $\check{A}_{\rho, r, n}(z)$ and $\check{B}_{\rho, r, n}(z)$.

We now claim that $(1-z)^{n+1}$ also divides $\check{C}_{r, n}(z)$. To prove this, we use the integral representation for $S_{r, n}(z)$ given by Lemma 4.4. Differentiating $n+1$ times under the integral, we obtain

$$
\widehat{S}_{r, n}(z)=(n+1)(-z)^{n+1} \int_{0}^{1} \frac{P_{r, n}(x)}{(z-x)^{n+2}} \mathrm{~d} x .
$$

Again by Lemma 4.3 and the Padé conditions at $z=1$ in $\mathcal{P}_{r, n}$ for $U_{j, r, n}$ and $V_{j, r, n}$, we deduce that

$$
P_{r, n}(x)=\mathcal{O}\left((1-x)^{n+1}\left(1+|\log (1-x)|^{2 r+1}\right)\right)
$$

as $x \rightarrow 1, x<1$. Therefore the singularity of $\widehat{S}_{r, n}(z)$ at $z=1$ is at most a power of logarithm. The expression (5.1) for $\widehat{S}_{r, n}(z)$, together with the above deductions made for $\check{A}_{\rho, r, n}(z)$ and $\check{B}_{\rho, r, n}(z)$, implies the claim.

We can summarize the above results as follows: there exist polynomials $\widehat{A}_{\rho, r, n}(z), \widehat{B}_{\rho, r, n}(z)(\rho \in\{0, \ldots, r\})$ and $\widehat{C}_{r, n}(z)$, all of degree at most $n$, such that

$$
\begin{aligned}
\widehat{S}_{r, n}(z)= & \sum_{\rho=0}^{r}\left[\widehat{A}_{\rho, r, n}(z) \operatorname{Li}_{\{1\}_{2 \rho+2}}^{\{\ell s\}_{\rho} \ell}\left(\frac{1}{z}\right)\right. \\
& \left.+\widehat{B}_{\rho, r, n}(z) \operatorname{Li}_{\{1\}_{2 \rho+1}}^{\{s \ell\}_{\rho}}\left(\frac{1}{z}\right)\right]+\widehat{C}_{r, n}(z)=\mathcal{O}\left(\frac{1}{z^{(r+1)(n+1)}}\right) .
\end{aligned}
$$

\subsection{Second reduction}

We want to find further Padé conditions involving the polynomials $\widehat{A}_{\rho, r, n}(z)$, $\widehat{B}_{\rho, r, n}(z)(\rho \in\{0, \ldots, r\})$ and $\widehat{C}_{r, n}(z)$. For this, we form the functions

$$
\begin{aligned}
Q_{j, r, n}:=\sum_{\rho=j}^{r}[ & -A_{\rho, r, n}(z) \operatorname{Li}_{2\{1\}_{2 \rho-2 j}}^{\{s \ell\}_{\rho-j}}(z)+B_{\rho, r, n}(z) \operatorname{Li}_{\{1\}_{2 \rho-2 j+1}}^{\{s \ell\}_{\rho-j}}(z) \\
& \left.+C_{\rho, r, n}(z) \mathrm{Li}_{\{1\}_{2 \rho-2 j}}^{\{\ell s\}_{\rho-j-1} \ell}(z)\right]
\end{aligned}
$$

where $j=0, \ldots, r$, and $A_{\rho, r, n}(z), B_{\rho, r, n}(z), C_{\rho, r, n}(z)$ are the polynomials in our initial Padé problem $\mathcal{P}_{r, n}$. Each $Q_{j, r, n}(z)$ is holomorphic at $z=0$ and the rules of 
differentiation of multiple polylogarithms (see Section 3.1) show that

$$
\begin{aligned}
\widehat{Q}_{j, r, n}(z) & :=\frac{z^{n+1}}{n !} Q_{j, r, n}^{(n+1)}(z) \\
& =\sum_{\rho=j}^{r}\left[\widehat{A}_{\rho, r, n}(z) \mathrm{Li}_{\{1\}_{2 \rho-2 j+1}}^{\{s \ell\}_{\rho-j}}(z)+\widehat{B}_{\rho, r, n}(z) \mathrm{Li}_{\{1\}_{2 \rho-2 j}}^{\{\ell s\}_{\rho-j-1} \ell}(z)\right]=\mathcal{O}\left(z^{n+1}\right)
\end{aligned}
$$

for all $j=0, \ldots r$. The main point here is that the polynomials $\widehat{A}_{\rho, r, n}(z)$ and $\widehat{B}_{\rho, r, n}(z)$ are the same as in Equation (5.4).

\subsection{The intermediate Padé problem $\mathcal{Q}_{r, n}$}

The previous two sections show that any solution $S_{r, n}(z)$ to the problem $\mathcal{P}_{r, n}$ yields (by differentiating $n+1$ times and multiplying by $z^{n+1} / n$ !) a solution to the following problem: given non-negative integers $r$ and $n$, find polynomials $\widehat{A}_{\rho, r, n}(z)$, $\widehat{B}_{\rho, r, n}(z)$ (for $\left.0 \leq \rho \leq r\right)$ and $\widehat{C}_{r, n}(z)$, of degrees $\leq n$, such that the following holds:

$$
\begin{aligned}
\widehat{S}_{r, n}(z):= & \sum_{\rho=0}^{r}\left[\widehat{A}_{\rho, r, n}(z) \mathrm{Li}_{\{1\}_{2 \rho+2}}^{\{\ell s\}_{\rho} \ell}\left(\frac{1}{z}\right)+\widehat{B}_{\rho, r, n}(z) \mathrm{Li}_{\{1\}_{2 \rho+1}}^{\{s \ell\}_{\rho}}\left(\frac{1}{z}\right)\right] \\
& +\widehat{C}_{r, n}(z)=\mathcal{O}\left(\frac{1}{z^{(r+1)(n+1)}}\right), \\
\widehat{Q}_{j, r, n}(z):= & \sum_{\rho=j}^{r}\left[\widehat{A}_{\rho, r, n}(z) \mathrm{Li}_{\{1\}_{2 \rho-2 j+1}}^{\{s\}_{\rho-j}}(z)+\widehat{B}_{\rho, r, n}(z) \mathrm{Li}_{\{1\}_{2 \rho-2 j}}^{\{\ell s\}_{\rho-j-1} \ell}(z)\right] \\
= & \mathcal{O}\left(z^{n+1}\right), \quad j=0, \ldots, r .
\end{aligned}
$$

We shall denote this Padé approximation problem by $\mathcal{Q}_{r, n}$. It amounts to solving a linear system of $2(r+1)(n+1)-1$ equations in $2(r+1)(n+1)$ unknowns (the coefficients of the polynomials $\widehat{A}_{\rho, r, n}(z)$ and $\left.\widehat{B}_{\rho, r, n}(z)\right)$. Hence it has at least one non trivial solution and our next task is to prove that is has exactly one solution up to a multiplicative constant.

To do so, we will identify the problem with one already solved by Sorokin [20]. We first observe the effect of changing $z$ to $1-z$ in the Padé problem $\mathcal{Q}_{r, n}$.

Lemma 5.1. For any $z \in \mathbb{C} \backslash[0,1]$, we have

$$
\begin{aligned}
\mathrm{Li}_{\{1\}_{2 \rho+1}}^{\{s \ell\}_{\rho}}\left(\frac{1}{z}\right) & =(-1)^{\rho+1} \operatorname{Li}_{1\{2\}_{\rho}}{ }_{\{s\}_{\rho}}\left(\frac{1}{1-z}\right), \\
\operatorname{Li}_{\{1\}_{2 \rho+2}}^{\{\ell s\}_{\rho} \ell}\left(\frac{1}{z}\right) & =(-1)^{\rho+1} \operatorname{Li}_{\{2\}_{\rho+1}}^{\{s\}_{\rho}}\left(\frac{1}{1-z}\right) .
\end{aligned}
$$


Proof. We prove these identities by induction on $\rho$. They hold trivially for $\rho=0$ and by differentiation of both sides at level $\rho$, we get the identity at level $\rho-1$. We deduce that the identity at level $\rho$ holds, up to some additive constant. This constant must be 0 because both sides vanish at $z=\infty$.

Therefore, when we change $z$ to $1-z$, the Padé problem $\mathcal{Q}_{r, n}$ becomes

$$
\begin{aligned}
\widehat{S}_{r, n}(1-z):= & \sum_{\rho=0}^{r}(-1)^{\rho+1}\left[\widehat{A}_{\rho, r, n}(1-z) \operatorname{Li}_{\{2\}_{\rho+1}}^{\{s\}_{\rho}}\left(\frac{1}{z}\right)\right. \\
& \left.+\widehat{B}_{\rho, r, n}(1-z) \operatorname{Li}_{1\{2\}_{\rho}}^{\{s\}_{\rho}}\left(\frac{1}{z}\right)\right] \\
& +\widehat{C}_{r, n}(1-z)=\mathcal{O}\left(\frac{1}{(1-z)^{(r+1)(n+1)}}\right)=\mathcal{O}\left(\frac{1}{z^{(r+1)(n+1)}}\right) \\
\widehat{Q}_{j, r, n}(1-z):= & \sum_{\rho=j}^{r}\left[\widehat{A}_{\rho, r, n}(1-z) \operatorname{Li}_{\{1\}_{2 \rho-2 j+1}}^{\{s \ell\}_{\rho-j}}(1-z)\right. \\
\left.\quad+\widehat{B}_{\rho, r, n}(1-z) \operatorname{Li}_{\{1\}_{2 \rho-2 j}}^{\{\ell s\}_{\rho-j-1} \ell}(1-z)\right] & \\
= & \mathcal{O}\left((1-z)^{n+1}\right), \quad j=0, \ldots, r .
\end{aligned}
$$

Let us define

$$
\begin{aligned}
\widetilde{A}_{\rho, r, n}(z) & =(-1)^{\rho+1} \widehat{A}_{\rho, r, n}(1-z), \quad \widetilde{B}_{\rho, r, n}(z)=(-1)^{\rho+1} \widehat{B}_{\rho, r, n}(1-z), \\
\widetilde{C}_{r, n}(z) & =\widehat{C}_{r, n}(1-z), \quad \widetilde{S}_{r, n}(z)=\widehat{S}_{r, n}(1-z), \quad \widetilde{Q}_{j, r, n}(z)=-\widehat{Q}_{j, r, n}(1-z) .
\end{aligned}
$$

With this notation, the Padé problem $\mathcal{Q}_{r, n}$ now reads

$$
\begin{aligned}
\widetilde{S}_{r, n}(z) & :=\sum_{\rho=0}^{r}\left[\widetilde{A}_{\rho, r, n}(z) \operatorname{Li}_{\{2\}_{\rho+1}}\left(\frac{s\}_{\rho}}{z}\right)+\widetilde{B}_{\rho, r, n}(z) \operatorname{Li}_{1\{2\}_{\rho}}^{\{s\}_{\rho}}\left(\frac{1}{z}\right)\right]+\widetilde{C}_{r, n}(z) \\
& =\mathcal{O}\left(\frac{1}{z^{(r+1)(n+1)}}\right) \\
\widetilde{Q}_{j, r, n}(z) & :=\sum_{\rho=j}^{r}(-1)^{\rho}\left[\widetilde{A}_{\rho, r, n}(z) \operatorname{Li}_{\{1\}_{2 \rho-2 j+1}}(1-z)+\widetilde{B}_{\rho, r, n}(z) \operatorname{Li}_{\{1\}_{2 \rho-2 j}}^{\{\ell s\}_{\rho-j-1} \ell}(1-z)\right] \\
& =\mathcal{O}\left((1-z)^{n+1}\right), \quad j=0, \ldots, r .
\end{aligned}
$$

In spite of different notation, we recognize here Sorokin's problem [20] for $\pi^{2}$ of weight $2 r+2$, which we denote by $\mathcal{R}_{r, n}$ from now on. Sorokin proved that this problem has a unique solution up to proportionality. Therefore the same property holds for $\mathcal{Q}_{r, n}$, and also for $\mathcal{P}_{r, n}$. This concludes the proof of Theorem 1.1, except for the integral representation (1.3) of $S_{r, n}(z)$ that we shall prove now. 


\subsection{Hypergeometric integrals for $\tilde{S}_{r, n}(z)$ and $S_{r, n}(z)$}

Sorokin has found an explicit integral formula for the solution $\widetilde{S}_{r, n}(z)$ of his Padé problem $\mathcal{R}_{r, n}$ stated in Section 5.3 (see [20, Lemma 17, page 1835]), namely

$$
\widetilde{S}_{r, n}(z)=(-1)^{(r+1) n} \int_{[0,1]^{2 r+2}} \prod_{j=1}^{r+1} \frac{x_{j}^{n}\left(1-x_{j}\right)^{n} y_{j}^{n}\left(1-y_{j}\right)^{n}}{\left(\frac{z}{x_{1} y_{1} \cdots x_{j-1} y_{j-1}}-x_{j} y_{j}\right)^{n+1}} \mathrm{~d} x_{j} \mathrm{~d} y_{j}
$$

In this and the next subsections we shall deduce from it the integral expression (1.3) of $S_{r, n}(z)$, using the relation

$$
\frac{z^{n+1}}{n !} S_{r, n}^{(n+1)}(z)=\widetilde{S}_{r, n}(1-z)
$$

and the integral operator defined in Subsection 3.2.

To begin with, we recall that Sorokin solved his Padé approximation problem $\mathcal{R}_{r, n}$ recursively and showed that, for any integer $r \geq 1$ and any $z \in \mathbb{C} \backslash[0,1]$,

$$
\tilde{S}_{r-1, n}(z)=\frac{1}{n !^{2}} z^{n+1}(1-z)^{n+1}\left(z^{n+1} \tilde{S}_{r, n}^{(n+1)}(z)\right)^{(n+1)}
$$

and

$$
\tilde{S}_{0, n}(z)=\int_{0}^{1} \int_{0}^{1} \frac{x^{n}(1-x)^{n} y^{n}(1-y)^{n}}{(z-x y)^{n+1}} \mathrm{~d} x \mathrm{~d} y .
$$

It is not hard to see that, with the notation of Subsection 3.2, we have for $z \in$ $\mathbb{C} \backslash[0,1]:$

$$
\tilde{S}_{0, n}(z)=\mathbf{H}_{n+1,0}^{n+1}\left(\int_{0}^{1} \frac{x^{n}(1-x)^{n}}{(z-x)^{n+1}} \mathrm{~d} x\right)=\mathbf{H}_{n+1,0}^{n+1} \mathbf{H}_{n+1, n+1}^{n+1}(\mathbf{1}),
$$

where $\mathbf{1}$ is the constant function equal to 1 on $\mathbb{C} \backslash[0,1]$. We can apply the general properties of hypergeometric integrals proved in Subsection 3.2 to (5.7) and we get the following result, which is nothing but (5.5) written in a different language (see Subsection 5.5 for details). We recall that $f^{\partial}(z):=f(1-z)$ and we denote by $\mathbf{H}^{k}=\mathbf{H} \circ \mathbf{H} \circ \cdots \circ \mathbf{H}$ the composition of an integral operator $\mathbf{H}$ with itself $k$ times.

Proposition 5.2. For any $z \in \mathbb{C} \backslash[0,1]$ and any integer $r \geq 0$, we have

$$
\tilde{S}_{r, n}(z)=\left(\mathbf{H}_{n+1,0}^{n+1} \mathbf{H}_{n+1, n+1}^{n+1}\right)^{r+1}(\mathbf{1})(z)
$$

and

$$
\tilde{S}_{r, n}^{\partial}(z)=\left(\mathbf{H}_{0, n+1}^{n+1} \mathbf{H}_{n+1, n+1}^{n+1}\right)^{r+1}(\mathbf{1})(z) .
$$


Equation (5.9) follows immediately from Equation (5.8) and the relation

$$
\tilde{S}_{r, n}=\mathbf{H}_{n+1,0}^{n+1} \mathbf{H}_{n+1, n+1}^{n+1}\left(\tilde{S}_{r-1, n}\right)
$$

which is just a translation of Equation (5.7) (using Lemma 3.3). Then Equation (5.10) follows from (5.9) by means of Lemma 3.6. Now Equation (5.6) reads

$$
\frac{z^{n+1}}{n !} S_{r, n}^{(n+1)}(z)=\tilde{S}_{r, n}^{\partial}(z)
$$

and $\lim _{z \rightarrow \infty} S_{r, n}(z)=0$ for any $r \geq 0$, so that Lemma 3.3 yields

$$
S_{r, n}(z)=\mathbf{H}_{n+1,0}^{n+1}\left(\tilde{S}_{r, n}^{\partial}\right)(z)
$$

Hence, by (5.10) in Proposition 5.2, we obtain the following result (using also Lemma 3.5 to take limits as $z \rightarrow 1$ ):

Proposition 5.3. For any $z \in \mathbb{C} \backslash[0,1]$ and any integer $r \geq 0$, we have

$$
S_{r, n}(z)=\mathbf{H}_{n+1,0}^{n+1}\left(\mathbf{H}_{0, n+1}^{n+1} \mathbf{H}_{n+1, n+1}^{n+1}\right)^{r+1}(\mathbf{1})(z) .
$$

Moreover, both sides of (5.12) are defined and equal for $z=1$.

\subsection{Explicit multiple integrals}

The integral expression for $S_{r, n}(z)$ given in Theorem 1.1 is simply the explicit "expansion" of the formula (5.12) given in Proposition 5.3 above. Let us provide details on this expansion.

For any function $F$ analytic on $\mathbb{C} \backslash[0,1]$ and at infinity, Equation (3.1) in Subsection 3.2 reads

$$
\mathbf{H}_{n+1, n+1}^{n+1}(F)(z)=(-1)^{n+1} \int_{0}^{1} \frac{u^{n}(1-u)^{n}}{(u-z)^{n+1}} F\left(\frac{z}{u}\right) \mathrm{d} u .
$$

This function $\mathbf{H}_{n+1, n+1}^{n+1}(F)(z)$ is analytic on $\mathbb{C} \backslash[0,1]$ and at infinity, and vanishes to an order $\geq n+1$ at $\infty$ (using Lemma 3.3). The same property can be proved in the same way for the following function:

$$
\begin{aligned}
\mathbf{H}_{0, n+1}^{n+1} \mathbf{H}_{n+1, n+1}^{n+1}(F)(z) & =z^{n+1} \int_{0}^{1} \frac{v^{-1}(1-v)^{n}}{(v-z)^{n+1}} \int_{0}^{1} \frac{u^{n}(1-u)^{n}}{(u-z / v)^{n+1}} F\left(\frac{z}{u v}\right) \mathrm{d} u \mathrm{~d} v \\
& =z^{n+1} \int_{0}^{1} \int_{0}^{1} \frac{v^{n}(1-v)^{n} u^{n}(1-u)^{n}}{(v-z)^{n+1}(u v-z)^{n+1}} F\left(\frac{z}{u v}\right) \mathrm{d} u \mathrm{~d} v
\end{aligned}
$$


By induction on $r \geq 0$ this implies, using Equation (5.10):

$$
\begin{aligned}
& \tilde{S}_{r, n}^{\partial}(z)=\left(\mathbf{H}_{0, n+1}^{n+1} \mathbf{H}_{n+1, n+1}^{n+1}\right)^{r+1}(\mathbf{1})(z)=z^{(r+1)(n+1)} \\
& \quad \times \int_{[0,1]^{2(r+1)}} \frac{\prod_{j=1}^{r+1}\left(\left(u_{j} v_{j}\right)^{(r-j+2)(n+1)-1}\left(1-u_{j}\right)^{n}\left(1-v_{j}\right)^{n}\right)}{\prod_{j=1}^{r+1}\left(\left(z-u_{1} v_{1} \cdots u_{j-1} v_{j-1} u_{j}\right)^{n+1}\left(z-u_{1} v_{1} \cdots u_{j} v_{j}\right)^{n+1}\right)} \mathrm{dud} \mathbf{v} .
\end{aligned}
$$

Therefore the equality

$$
\mathbf{H}_{n+1,0}^{n+1}\left(\mathbf{H}_{0, n+1}^{n+1} \mathbf{H}_{n+1, n+1}^{n+1}\right)^{r+1}(\mathbf{1})(z)=(-1)^{n+1} \int_{0}^{1} u_{0}^{-1}\left(1-u_{0}\right)^{n} \tilde{S}_{r, n}^{\partial}\left(z / u_{0}\right) \mathrm{d} u_{0}
$$

yields, using Proposition 5.3:

$$
\begin{aligned}
& S_{r, n}(z)=(-1)^{n+1} z^{(r+1)(n+1)} \\
& \times \int_{[0,1]^{2 r+3}} \frac{u_{0}^{(r+1)(n+1)-1}\left(1-u_{0}\right)^{n} \prod_{j=1}^{r+1}\left(\left(u_{j} v_{j}\right)^{(r-j+2)(n+1)-1}\left(1-u_{j}\right)^{n}\left(1-v_{j}\right)^{n}\right)}{\prod_{j=1}^{r+1}\left(\left(z-u_{0} u_{1} v_{1} \cdots u_{j-1} v_{j-1} u_{j}\right)^{n+1}\left(z-u_{0} u_{1} v_{1} \cdots u_{j} v_{j}\right)^{n+1}\right)} \mathrm{dud} \mathbf{v} .
\end{aligned}
$$

This completes the proof of Theorem 1.1.

\section{Beyond Vasilyev's conjecture: irrationality of odd zeta values}

A natural problem is to find a proof that the numbers $\zeta(2 r+1)$, for $r \geq 1$, span an infinite-dimensional $\mathbb{Q}$-vector space $[2,16]$ that would be analogous to Sorokin's proof that $\pi$ is transcendental [20] (since Sorokin's result is equivalent to the fact that the numbers $\zeta(2 r)$, for $r \geq 1$, span an infinite-dimensional $\mathbb{Q}$-vector space). In particular, such a proof would involve a Padé approximation problem with multiple polylogarithms.

Let $\sigma$ be an integer such that $1 \leq \sigma \leq r+2$. To achieve this goal, it is enough to relate the very-well-poised hypergeometric series

$$
\sum_{k=1}^{\infty}\left(k+\frac{n}{2}\right) \frac{(k-\sigma n)_{\sigma n}(k+n+1)_{\sigma n}}{(k)_{n+1}^{2 r+4}}
$$

which can be used to prove the above mentioned result (see for instance [8]), to such a Padé approximation problem. An analogous work has been done in [10], 
where this series is related to a Padé approximation problem involving only classical polylogarithms, namely of depth 1 .

We shall prove now that for $\sigma=1$ the hypergeometric series (6.1) is equal (up to a sign) to $S_{r, n}(1)$, thereby providing in this case the relation we are looking for. For any $\sigma$ we shall prove that this series is the value at $z=1$ of a function $S_{r, n, \sigma}(z)$ which generalizes $S_{r, n}(z)$; what is missing is a Padé approximation problem of which $S_{r, n, \sigma}(z)$ would be a solution. We believe that a suitable generalisation of the problem $\mathcal{P}_{r, n}$ solved in Theorem 1.1 could have this property.

With this aim in view, we consider the function $S_{r, n, \sigma}(z)$ defined by

$$
\frac{z^{n+1}}{n !} S_{r, n, \sigma}^{(\sigma n+1)}(z)=\tilde{S}_{r, n}^{\partial}(z)
$$

and $\lim _{z \rightarrow \infty} S_{r, n, \sigma}(z)=0$; in this way we have $S_{r, n, 1}(z)=S_{r, n}(z)$ (see Equation (5.11)). We have

$$
S_{r, n, \sigma}(z)=\mathbf{H}_{n+1,0}^{\sigma n+1}\left(\widetilde{S}_{r, n}^{\partial}\right)(z) .
$$

The equality

$$
\begin{aligned}
& \mathbf{H}_{n+1,0}^{\sigma n+1}\left(\mathbf{H}_{0, n+1}^{n+1} \mathbf{H}_{n+1, n+1}^{n+1}\right)^{r+1}(\mathbf{1})(z) \\
& =(-1)^{\sigma n+1} z^{(\sigma-1) n} \int_{0}^{1} u_{0}^{(1-\sigma) n-1}\left(1-u_{0}\right)^{\sigma n} \tilde{S}_{r, n}^{\partial}\left(z / u_{0}\right) \mathrm{d} u_{0}
\end{aligned}
$$

yields, using Proposition 5.3:

$$
\begin{aligned}
& S_{r, n, \sigma}(z)=(-1)^{\sigma n+1} z^{(r+\sigma) n+r+1} \\
& \times \int_{[0,1]^{2 r+3}} \frac{u_{0}^{(r-\sigma+2) n+r}\left(1-u_{0}\right)^{\sigma n} \prod_{j=1}^{r+1}\left(\left(u_{j} v_{j}\right)^{(r-j+2)(n+1)-1}\left(1-u_{j}\right)^{n}\left(1-v_{j}\right)^{n}\right)}{\prod_{j=1}^{r+1}\left(\left(z-u_{0} u_{1} v_{1} \cdots u_{j-1} v_{j-1} u_{j}\right)^{n+1}\left(z-u_{0} u_{1} v_{1} \cdots u_{j} v_{j}\right)^{n+1}\right)} \mathrm{dud} \mathbf{v} .
\end{aligned}
$$

This function has the following value at $z=1$ :

$$
\begin{aligned}
& S_{r, n, \sigma}(1)=(-1)^{\sigma n+1} \\
& \times \int_{[0,1]^{2 r+3}} \frac{u_{0}^{(r-\sigma+2) n+r}\left(1-u_{0}\right)^{\sigma n} \prod_{j=1}^{r+1}\left(\left(u_{j} v_{j}\right)^{(r-j+2)(n+1)-1}\left(1-u_{j}\right)^{n}\left(1-v_{j}\right)^{n}\right)}{\left.\prod_{j=1}^{r+1}\left(1-u_{0} u_{1} v_{1} \cdots u_{j-1} v_{j-1} u_{j}\right)^{n+1}\left(1-u_{0} u_{1} v_{1} \cdots u_{j} v_{j}\right)^{n+1}\right)} \mathrm{dud} \mathbf{v} .
\end{aligned}
$$

Using Proposition 17 of [7] (which amounts to a change of variables) one obtains

$$
S_{r, n, \sigma}(1)=(-1)^{\sigma n+1} \int_{[0,1]^{a-1}} \frac{\prod_{j=1}^{a-1} x_{j}^{\sigma n}\left(1-x_{j}\right)^{n}}{\left(1-x_{1} x_{2} \cdots x_{a-1}\right)^{\sigma n+1} \prod_{\substack{2 \leq j \leq a-2 \\ j \text { even }}}\left(1-x_{1} x_{2} \cdots x_{j}\right)^{n+1}} \mathrm{~d} \mathbf{x}
$$


with $a=2 r+4$. Then using Zlobin's result [23] or another change of variables (namely Théorème 10 of [7]), one obtains the Vasilyev-type integral

$$
S_{r, n, \sigma}(1)=(-1)^{\sigma n+1} \int_{[0,1]^{a-1}} \frac{\prod_{j=1}^{a-1} x_{j}^{\sigma n}\left(1-x_{j}\right)^{n}}{Q_{a-1}\left(x_{1}, \cdots, x_{a-1}\right)^{\sigma n+1}} \mathrm{~d} \mathbf{x} .
$$

Now Theorem 5 of [25] yields

$$
S_{r, n, \sigma}(1)=(-1)^{\sigma n+1} \sum_{k=1}^{\infty}\left(k+\frac{n}{2}\right) \frac{(k-\sigma n)_{\sigma n}(k+n+1)_{\sigma n}}{(k)_{n+1}^{a}} .
$$

Up to a sign, this is exactly the very-well poised hypergeometric series (6.1).

\section{References}

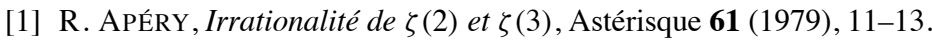

[2] K. M. BALL and T. RIVOAL, Irrationalité d'une infinité de valeurs de la fonction zêta aux entiers impairs, Invent. Math. 146 (2001), 193-207.

[3] F. BEuKERs, A note on the irrationality of $\zeta(2)$ and $\zeta(3)$, Bull. London Math. Soc. 11 (1979), 268-272.

[4] J. CResson, S. Fischler and T. RivoAL, Séries hypergéométriques multiples et polyzêetas, Bull. Soc. Math. France 136 (2008), 97-145.

[5] J. Cresson, S. Fischler and T. Rivone, Phénomènes de symétrie dans des formes linéaires en polyzêtas, J. Reine Angew. Math. 617 (2008), 109-152.

[6] S. FISCHLER, Formes linéaires en polyzêtas et intégrales multiples, C. R. Acad. Sci. Paris 335 (2002), 1-4.

[7] S. FISCHLER, Groupes de Rhin-Viola et intégrales multiples, J. Théor. Nombres Bordeaux 15 (2003), 479-534.

[8] S. FISCHLER, Irrationalité de valeurs de zêta (d'après Apéry, Rivoal, ...), Séminaire Bourbaki 2002-2003, exposé no. 910 (Nov. 2002), Astérisque 294 (2004), 27-62.

[9] S. FISCHLER, Multiple series connected to Hoffman's conjecture on multiple zeta values, J. Algebra 320 (2008), 1682-1703.

[10] S. FISCHLER and T. RIVOAL, Approximants de Padé et séries hypergéométriques équilibrées, J. Math. Pures Appl. 82 (2003), 1369-1394.

[11] P. Henrici, "Applied and Computational Complex Analysis", Vol. 2, Special functions, integral transforms, asymptotics, continued fractions, Wiley Classics Library, John Wiley \& Sons, Inc., New York, 1991.

[12] C. KrattenthaleR and T. Rivoal, An identity of Andrews, multiple integrals, and verywell-poised hypergeometric series, Ramanujan J. 13 (2007), 203-219.

[13] G. RHIN and C. ViOLA, On a permutation group related to $\zeta(2)$, Acta Arith. 77 (1996), 23-56.

[14] G. RHIN and C. Viola, The group structure for $\zeta(3)$, Acta Arith. 97 (2001), 269-293.

[15] G. RHIN and C. ViolA, Multiple integrals and linear forms in zeta-values, Funct. Approx. Comment. Math. 37 (2007), 429-444.

[16] T. RIVOAL, La fonction zêta de Riemann prend une infinité de valeurs irrationnelles aux entiers impairs, C. R. Acad. Sci. Paris 331 (2000), 267-270.

[17] T. RIVOAL, Linear forms in zeta values that arise from Sorokin-type integrals, Fundam. Prikl. Mat. 16 (2010), 161-172 (Russian). English translation in J. Math. Sci. (N.Y.) 180 (2012), 641-649. 
[18] A. B. ShidlovskiI, "Transcendental Numbers", de Gruyter Studies in Mathematics, Vol. 12, Walter de Gruyter \& Co., Berlin, 1989.

[19] V. N. Sorokin, Apéry's theorem, Vestnik Moskov. Univ. Ser. I Mat. Mekh. 53 (1998), 48-53 (Russian). English translation in Moscow Univ. Math. Bull. 53 (1998), 48-52.

[20] V. N. SOROKIn, A transcendence measure for $\pi^{2}$, Mat. Sbornik 187 (1996), 87-120 (Russian). English translation in Sb. Math. 187 (1996), 1819-1852.

[21] D. VASILYEV, Approximations of zero by linear forms in values of the Riemann zetafunction, Doklady Nat. Acad. Sci Belarus 45 (2001), 36-40 (Russian). Extended version in English : On small linear forms for the values of the Riemann zeta-function at odd points, preprint no. 1 (558), Nat. Acad. Sci. Belarus, Institute Math., Minsk (2001), 14 pages.

[22] C. ViOLA, On the equivalence of the Beukers and Sorokin multiple integrals, Fundam. Prikl. Mat. 16 (2010), 49-59 (Russian). English translation in J. Math. Sci. (N.Y.) 180 (2012), $561-568$.

[23] S. A. ZLOBIN, Integrals that can be represented as linear forms of generalized polylogarithms, Mat. Zametki 71 (2002), 782-787 (Russian). English translation in Math. Notes 71 (2002), 711-716.

[24] S. A. ZLobin, Generating functions for the values of a multiple zeta function, Vestnik Moskov. Univ. Ser. I Mat. Mekh. 73 (2005), 55-59 (Russian). English translation in Moscow Univ. Math. Bull. 60 (2005), 44-48.

[25] W. ZUDILIN, Well-poised hypergeometric service for Diophantine problems of zeta values, J. Théor. Nombres Bordeaux 15 (2003), 593-626.

[26] W. Zudilin, On the irrationality measure of $\pi^{2}$, Uspekhi Mat. Nauk 68 (2013), 171-172 (Russian). English translation in Russian Math. Surveys 68 (2013), 1133-1135.

Laboratoire de Mathématiques d'Orsay

Université Paris-Sud, CNRS

Université Paris-Saclay

91405 Orsay, France

stephane.fischler@math.u-psud.fr

Institut Fourier

CNRS et Université Grenoble 1

100 rue des maths, BP 74

38402 St Martin d'Hères Cedex, France

tanguy.rivoal@ujf-grenoble.fr 